\title{
Maternal smoking during pregnancy induces airway remodelling in mice offspring
}

\author{
M.J. Blacquière*, W. Timens*, B.N. Melgert*, M. Geerlings*, \\ D.S. Postma\# and M.N. Hylkema*
}

ABSTRACT: Children from smoking mothers have an increased risk of developing asthma for reasons largely unknown. The effects of maternal smoking during pregnancy on remodelling, allergic airway inflammation and hyperresponsiveness in offspring were investigated in an experimental asthma model.

Mice were exposed to fresh air or cigarette smoke from 3 weeks prior to conception until birth. Offspring were exposed to house dust mite (HDM) or PBS intranasally four times per week from week 5 to week 10 after birth onwards.

Maternal smoking increased airway smooth muscle layer, collagen III deposition and HDMinduced goblet cell numbers in offspring. It additionally increased methacholine responsiveness, which correlated significantly with increased airway smooth muscle layer and collagen deposition. Maternal smoking increased HDM-induced numbers of neutrophils and mast cells in lung tissue. No further effects were observed.

Smoking during pregnancy induces airway remodelling in mice offspring, which may contribute to increased methacholine responsiveness. This takes place irrespective of allergen exposure but may worsen the outcome of the allergic stimulus, resulting in higher methacholine responsiveness in house dust mite-exposed offspring from smoking mothers when compared to nonsmoking mothers. The results provide a possible mechanism behind the association between maternal smoking and asthma.

KEYWORDS: Airway remodelling, airway responsiveness, asthma, house dust mite, offspring, smoking during pregnancy

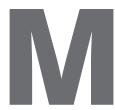

aternal smoking during pregnancy has been shown to increase physician-diagnosed asthma and wheezing during childhood, and some studies reported this to be independent of postnatal smoke exposure [1, 2]. In addition, two large studies in North America and several European countries have shown that maternal smoking during pregnancy is associated with reduced lung function [3, 4], and that maternal smoking during pregnancy is a strong and significant independent predictor of wheezing at age $>16$ yrs [5]. These studies mainly report effects in children and adolescents aged 0-18 yrs. Whether maternal smoking during pregnancy also affects the development of asthma in adulthood is not certain, but it increases this risk in smoking adolescents [6].

The mechanisms underlying the increased risk of development of asthma in infants exposed to maternal tobacco smoking in utero are largely unknown. Several studies have shown that maternal smoking during pregnancy has effects on immune system development [7-9], lung development and lung function in offspring.

With respect to lung development, in utero smoke exposure in rats decreased the number and increased the size of alveoli in the offspring [10], whereas in rhesus monkeys in utero exposure to nicotine increased collagen deposition around large airways and vessels [11]. Lambs exposed to maternal nicotine in the last trimester of fetal life developed lung function in a manner suggestive of smaller airways and/or smaller or stiffer lungs [12]. Furthermore, the inner airway wall thickness was higher in children from smoking versus nonsmoking mothers in children who died from sudden infant death syndrome; evidence for lung remodelling due to in utero smoke exposure in humans was also shown [13].

The current authors set out to further unravel the consequences of in utero cigarette smoke
AFFILIATIONS

Depts of *Pathology, and

\#Pulmonology, University Medical Center Groningen, University of Groningen, Groningen,

The Netherlands.

CORRESPONDENCE

M.N. Hylkema

Dept of Pathology

University Medical Center Groningen Hanzeplein 1

P.0. Box 196

9700 RB Groningen

The Netherlands

Fax: 31503619107

E-mail: m.n.hylkema@path.umcg.nl

Received:

August 222008

Accepted after revision:

December 012008

SUPPORT STATEMENT

This work was supported by a grant from the European Commission as part of GABRIEL (a multidisciplinary study to identify the genetic and environmental causes of asthma in the European Community, contract No. 018996).

STATEMENT OF INTEREST

None declared. 
exposure for susceptibility to the development of allergic airway inflammation by studying house dust mite (HDM)induced airway remodelling (smooth muscle layer thickness, collagen III deposition and goblet cell hyperplasia), inflammation (T-helper type 2 (Th2) cytokines and inflammatory cells) and methacholine $(\mathrm{MCh})$ responsiveness in adult offspring from smoking mothers.

\section{METHODS}

\section{Animals}

Female and male BALB/c mice, aged 8-10 weeks, were obtained from Harlan (Horst, the Netherlands). Standard food and water were provided ad libitum, lights were set to a 12/ $12 \mathrm{~h}$ light/dark cycle. All animal protocols were approved by the local committee on animal experimentation (University of Groningen, Groningen, the Netherlands) and were performed under strict governmental and international guidelines on animal experimentation.

\section{Cigarette smoke exposure}

Mainstream cigarette smoke was generated by the smoke exposure system of the Tobacco and Health Research Institute of the University of Kentucky (Lexington, KY, USA), which is similar to active smoking, as previously described [14]. Female mice were exposed to fresh air or two nose-only smoking sessions per day, 5 days per week. Smoking sessions started with two puffs from a 2R1 reference cigarette (University of Kentucky), and increased daily until 24 puffs per session were reached after 3 weeks. Then females were introduced pairwise to one male to induce conception. Smoke exposure remained constant during the total pregnancy. Mothers and their offspring were not exposed to cigarette smoke after offspring were born. 2R1 reference cigarettes were chosen because of high tar and nicotine content, and because it was possible to induce emphysema using these cigarettes [14].

\section{Antigen administration in offspring}

Offspring were housed with the mother until the end of the weaning period (3 weeks). Male and female pups were then separated (eight pups per cage). Offspring (eight female and eight male pups per group) were mildly anaesthetised (inhaled isoflurane with oxygen) and exposed to PBS or purified whole body HDM extract (Dermatophagoides pteronyssinus; Greer Laboratories, Lenoir, NC, USA) intranasally (25 $\mu \mathrm{g}$ in $10 \mu \mathrm{L}$ PBS) to induce allergic airway inflammation, four times per week from week 5 until week 10 after birth.

\section{Assessment of MCh responsiveness}

$\mathrm{MCh}$ responsiveness was assessed $24 \mathrm{~h}$ after the last allergen exposure by measuring enhanced pause (Penh) in conscious, spontaneously breathing animals using a whole-body plethysmography system (Buxco Electronics, Petersfield, UK) as described earlier by the current authors' group [15]. An advantage of using this technique is that it allows detailed immunohistochemistry of the lung tissue after this procedure within the same mouse.

\section{Tissue and blood collection}

Mice were sacrificed under anaesthesia at 10 weeks of age. Blood was taken by heart puncture. The two smallest right lung lobes were snap frozen for multiplex ELISA, the two largest right lung lobes were snap frozen for immunohistochemistry, the left lung lobe was formalin fixed and embedded in paraffin for immunohistochemistry.

\section{Cytokines}

Concentrations of interleukin (IL)-2, IL-4, IL-5, IL-6, IL-10, IL-13, IL-17, interferon (IFN)- $\gamma$ and tumour necrosis factor (TNF) were measured in homogenised lung tissue with a multiplex ELISA system (Lincoplex Systems, St Charles, MO, USA) on a Luminex 100 system using Starstation software (Applied Cytometry Systems, Sheffield, UK).

Eotaxin was determined using an ELISA kit (R\&D, Minneapolis, MN, USA), performed as described by the manufacturer.

\section{Histology}

Frozen lung sections were made in the caudal-cranial axis from the ventral end (the convex side) of the azygous and diaphragmatic lobe (fig. 1). Sections were made where small/intermediate airways were present and large cartilaginous airways were totally or largely absent in the sectional plane. Paraffin embedded lung sections were made crosssectionally halfway along left lobe, 45 degrees from the dorsalventral axis (to exclude the large cartilaginous bronchi close to the hilus). Sections were made when small/intermediate airways were present and large cartilaginous airways were totally or largely absent in this sectional plane.

Eosinophils and neutrophils were determined by staining $4-\mu \mathrm{m}$ cryosections of lung tissue for cyanide resistant endogenous peroxidase activity with diaminobenzidine (Sigma

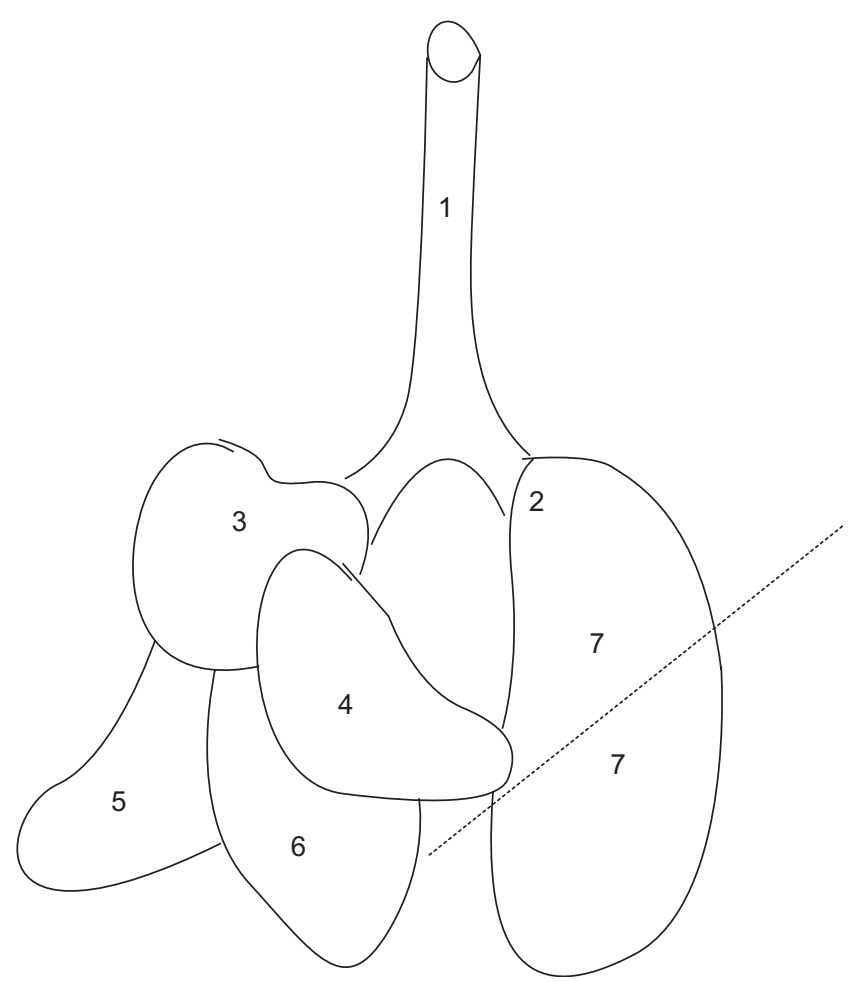

FIGURE 1. Lung lobe sampling. 1: trachea; 2: hylus; 3: apical lobe; 4: cardiac lobe; 5: azygous lobe; 6 : diaphragmatic lobe; 7 : left lobe. $\cdots \cdots \cdot$ : sectional plane. 

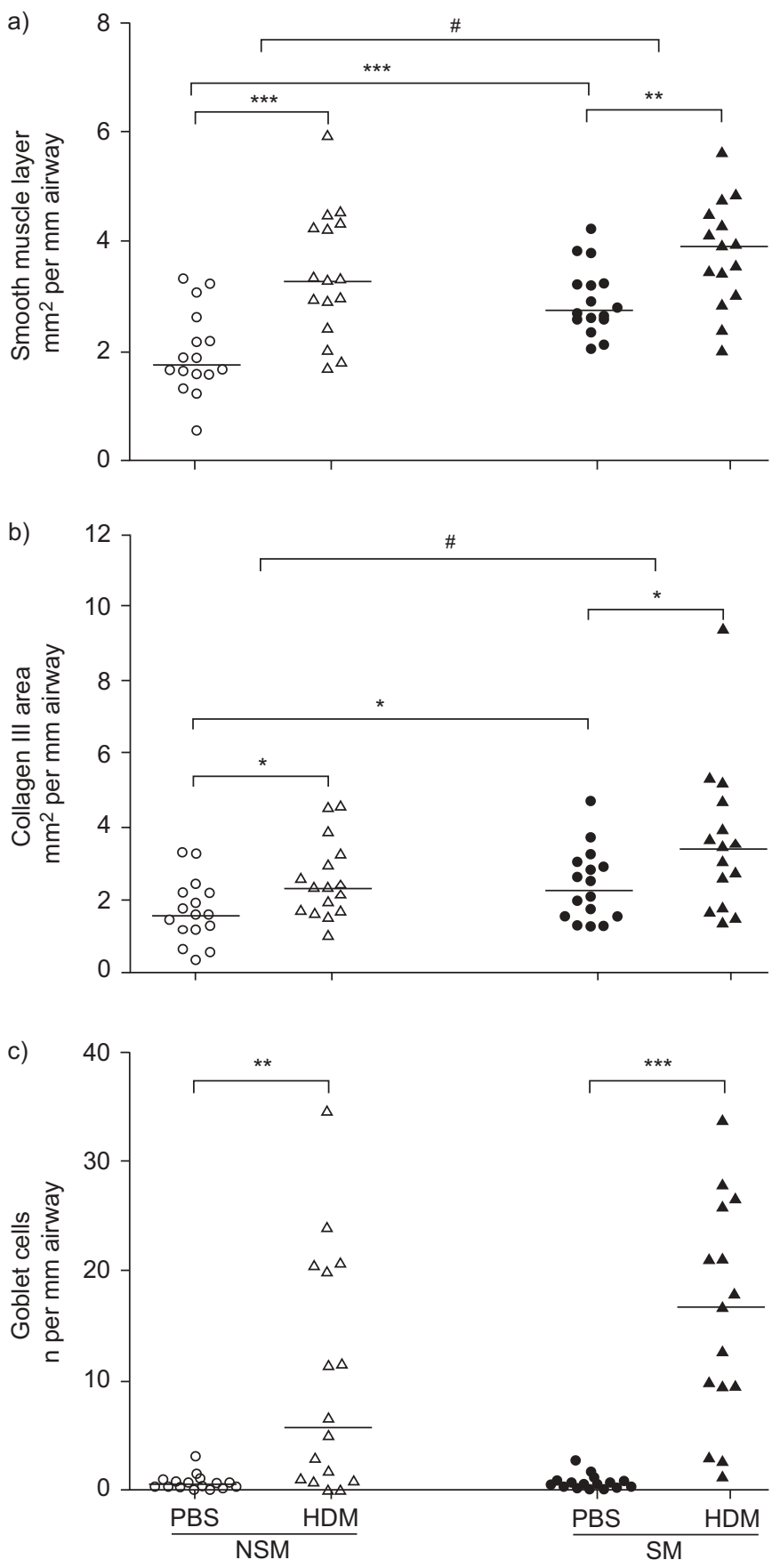

FIGURE 2. Remodelling in lung tissue from offspring exposed to PBS or house dust mite (HDM), from nonsmoking mothers (NSM) and smoking mothers (SM), expressed as: a) smooth muscle layer thickness around airways; b) collagen III deposition around airways; and c) goblet cell hyperplasia in airways. ${ }^{*}: \mathrm{p}<0.05 ;{ }^{* \star}: \mathrm{p}<0.01$; ${ }^{* \star *}: \mathrm{p}<0.001$; from subgroup analyses. ${ }^{*}: \mathrm{p}<0.01$; (SM effect); from linear regression analysis. a) HDM effect (NSM and SM groups): $p<0.001$ from linear regression analysis. b) HDM effect (NSM and SM groups): $p=0.002$ from linear regression analysis. c) Interaction SM and HDM effect: $p=0.038$ from linear regression analysis. "HDM effect" indicates a difference between both PBS-exposed groups versus both HDM-exposed groups. "SM effect" indicates a difference between both NSM groups versus both SM groups. "Interaction" indicates that the effect of HDM is greater in offspring from SM.
Aldrich, Zwijndrecht, the Netherlands) or a monoclonal rat anti GR1 antibody (BD Biosciences, San Jose, CA, USA) respectively. The volume percentage of eosinophils was calculated using morphometric analysis using Leica Qwin image analysis software (Leica Microsystems, Rijswijk, the Netherlands).

Mast cells were determined by staining 3- $\mu \mathrm{m}$ paraffinembedded sections of lung tissue with Toluidin Blue. Mast cells and neutrophils were counted manually in whole lung sections. The tissue area was quantified by morphometric analysis and the numbers of cells were expressed per $\mathrm{mm}^{2}$ lung tissue.

Goblet cells were stained with Periodic Acid Schiff's (PAS) in $3-\mu \mathrm{m}$ paraffin-embedded sections and all PAS-positive cells in the section were counted manually. The length of all airways (measured at the basal end of the airway epithelium) in the section was determined, and the total number of PAS-positive cells was expressed per mm airway.

Collagen III and airway smooth muscle layer were stained on $4-\mu \mathrm{m}$ frozen lung sections with polyclonal goat anti type-III collagen antibody (SBA, Birmingham, AL, USA) and a monoclonal mouse anti- $\alpha$-smooth muscle actin (anti- $\alpha$-SMA) antibody (Progen Biotechnik, Heidelberg, Germany), respectively. Collagen III and $\alpha$-SMA presence directly adjacent to the airway epithelium were quantified in the total lung section by morphometric analysis. The surface of positively stained tissue was expressed as $\mathrm{mm}^{2}$ per $\mathrm{mm}$ airway in the total lung section. Staining in the parenchyma and directly adjacent to blood vessels was excluded from the measurement.

Cartilaginous airways, if present, were excluded from all analyses. Each analysis was performed blinded by the same observer.

\section{Measurement of HDM-specific serum immunoglobulin E}

HDM-specific serum immunoglobulin (Ig)E was measured using ELISA. A flat-bottomed 96-well plate was coated overnight with rat-anti-mouse IgE (BD Biosciences). Serum samples were added (dilution 1:10) and incubated for $2 \mathrm{~h}$. Biotinylated HDM was added for $1 \mathrm{~h}$ and horseradish peroxidase-conjugated streptavidin (DAKO, Glostrup, Denmark) was added for $30 \mathrm{~min}$. Plates were developed using tetramethylbenzidine substrate (Sigma Aldrich), stopped, and optical densities were read at $450 \mathrm{~nm}$ using a Varioscan ELISA reader (Thermo Scientific, Breda, the Netherlands). HDM-specific IgE levels were expressed as arbitrary ELISA units by correcting optical densities for the dilution factor of the serum sample.

\section{Statistical analysis}

When residuals were not normally distributed, appropriate $\log _{10}$ or $1 / x$ transformation of the data was performed. The interaction of the effect of smoking during pregnancy and the effect of HDM exposure was investigated with a multiple linear regression model. The multiple linear regression analysis can distinguish between a positive interaction and a negative interaction. A significant positive interaction (the only interaction type found in the analyses) means that the effect of HDM exposure is greater in offspring from smoking mothers. 

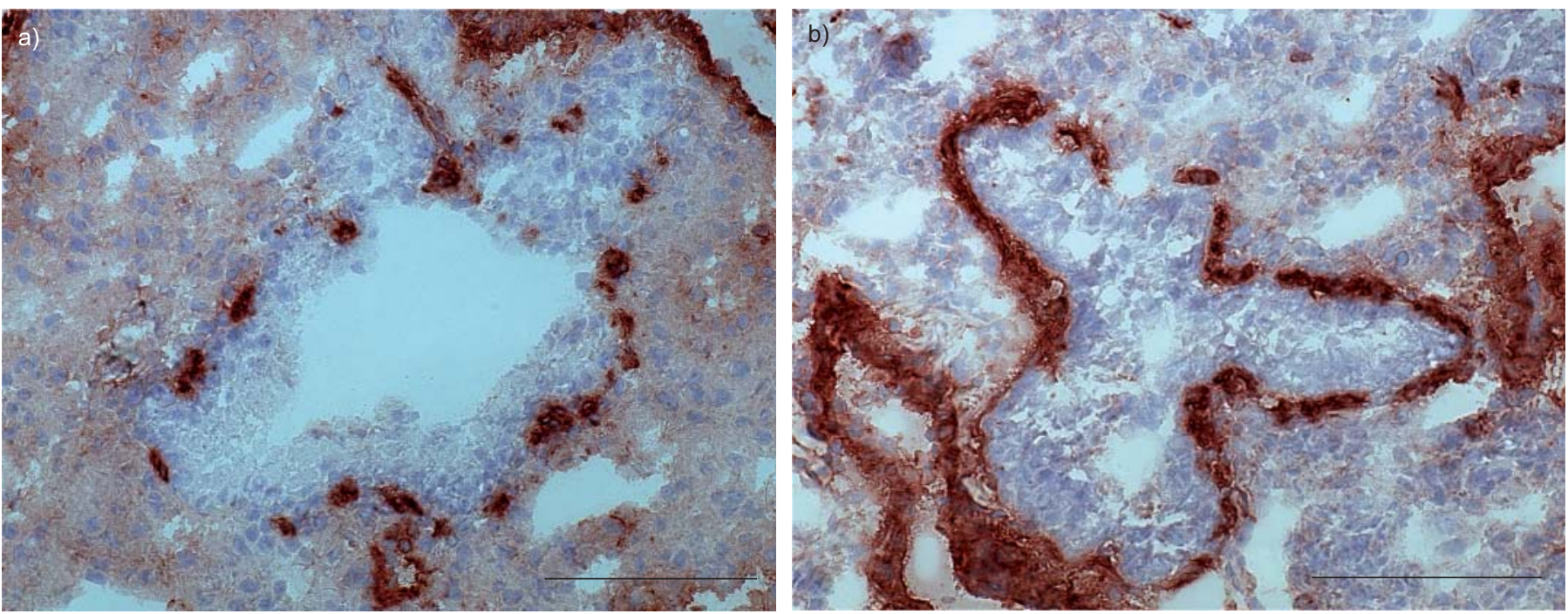

FIGURE 3. Immunohistochemical staining of airway smooth muscle (red) around airways in lung tissue from offspring from a) a nonsmoking mother and b) a smoking mother. Scale bars $=100 \mu \mathrm{m}$

When no interaction was found, the effect of smoking during pregnancy and the effect of HDM were assessed separately with linear regression analysis. To assess differences between subgroups, the normal distribution of the data in the subgroups was tested with a Kolmogorov-Smirnov and a Shapiro-Wilk test for normaility of data. When data were normally distributed according to these both tests, differences between subgroups were tested with a two-sided independent samples (unpaired) t-test. When data were not normally distributed two-sided Mann-Whitney U-tests were used.

Correlations between parameters were established using Spearman's rho. To further assess predictors of MCh responsiveness, parameters that were independently associated with the concentration of MCh inducing a 300\% increase in Penh (PC300) were identified and subsequently a stepwise linear regression model was used to explain the variability in PC300. A value of $p<0.05$ was considered significant.

\section{RESULTS}

\section{Maternal smoking during pregnancy induces airway remodelling in offspring}

Maternal smoking during pregnancy and HDM exposure independently increased the thickness of the airway smooth muscle layer (fig. 2a; fig. 3a and b) and deposition of collagen III around the airways from offspring (fig. $2 b$ ).

The number of goblet cells was analysed as an additional feature of airway remodelling. HDM exposure increased the number of goblet cells in the airways (fig. 2c), an effect that was stronger in offspring from smoking mothers than from nonsmoking mothers (indicated by a significant interaction between the effects of smoking during pregnancy and HDM exposure).

\section{Maternal smoking during pregnancy increases MCh responsiveness in offspring}

Exposure to HDM induced a significant increase in Penh in offspring from smoking and nonsmoking mothers, as calculated by the area under the Penh (a unitless index of airway hyperresponsiveness) curve (fig. 4a).

Maternal smoking during pregnancy increased $\mathrm{MCh}$ responsiveness in offspring, as shown by a decreased PC300 (fig. 4b). This increased MCh responsiveness was independent from HDM exposure. Offspring exposed to HDM had a lower PC300 than offspring exposed to PBS, which yields the lowest PC300 in HDM-exposed offspring from smoking mothers. PC300 correlated inversely with airway smooth muscle layer thickness (fig. 4c) and collagen III deposition (rho $-0.62, \mathrm{p}<0.001$ and rho $-0.41, \mathrm{p}=0.001$, respectively).

\section{Effect of maternal smoking during pregnancy on HDM- induced inflammatory cells and cytokines in offspring}

HDM exposure increased numbers of neutrophils and mast cells in lung tissue in offspring from smoking mothers, but not from nonsmoking mothers. HDM exposure increased eosinophils in lung tissue irrespective of maternal smoking (table 1).

Smoking during pregnancy had no effects on lung cytokines as investigated by linear regression (table 2). HDM exposure increased levels of IL-2, IL-4, IL-5, IL-6, IL-13, IL-17 and TNF in lung homogenate, whereas it decreased levels of IFN $-\gamma$ and IL-10. In particular, IL-17 was strongly upregulated after HDM exposure, which correlated with neutrophil (rho 0.51, $p=0.003$ ) and eosinophil numbers (rho 0.75, $p<0.001$ ) and eotaxin level (rho 0.61, $\mathrm{p}<0.001$ ) in lung tissue.

HDM-specific IgE levels in serum were increased after HDM exposure, without a significant effect of maternal smoking status (data not shown).

\section{Parameters explaining variability in MCh responsiveness in offspring}

In order to further assess whether the observed increased remodelling could underlie the increased MCh responsiveness, it was first identified whether smooth muscle layer thickness, collagen III deposition and goblet cell hyperplasia were independently associated with PC300. They were all independently 

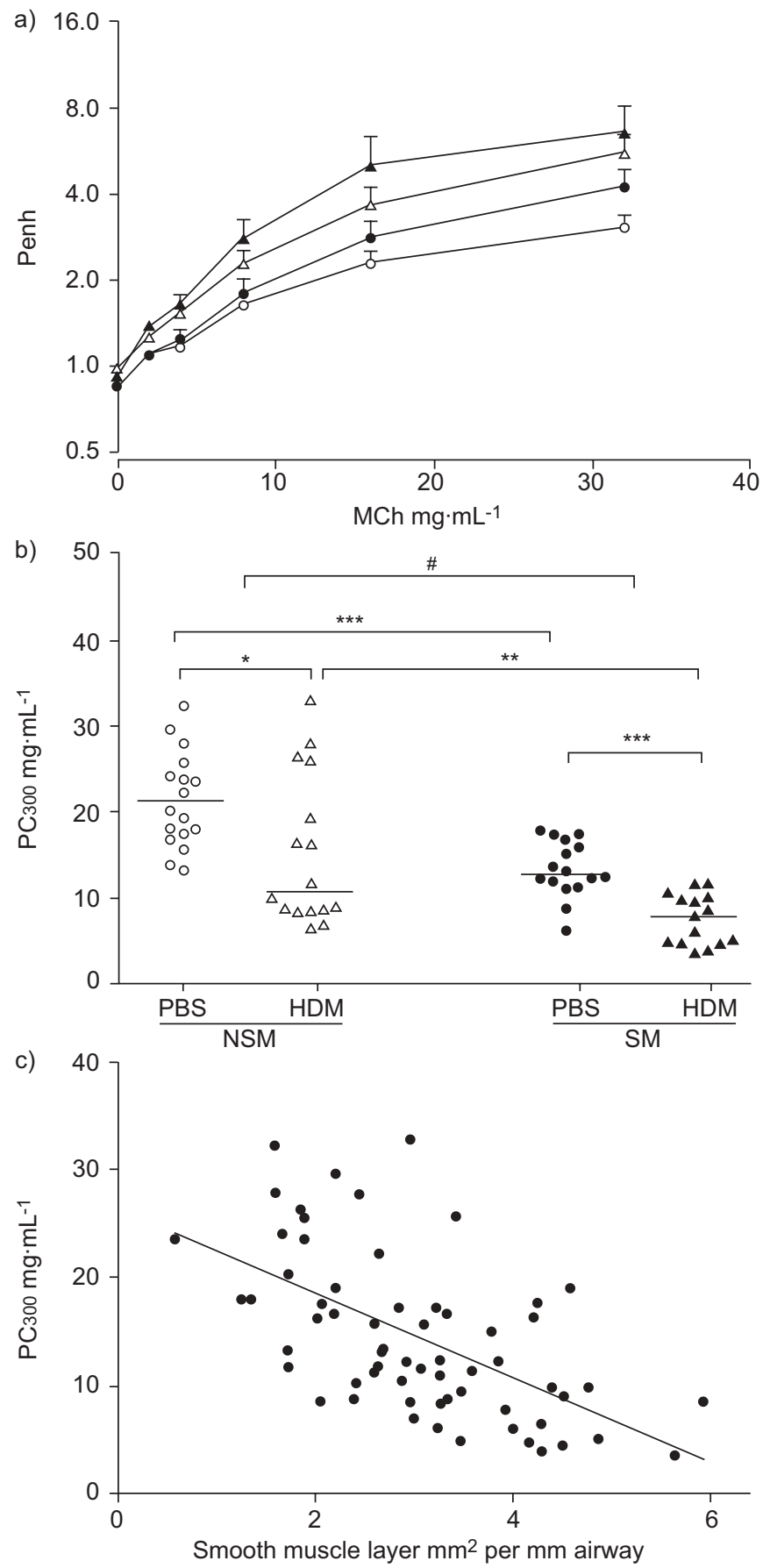

FIGURE 4. Methacholine (MCh) responsiveness from offspring exposed to PBS (circles) or house dust mite (HDM; triangles) from nonsmoking mothers (NSM open symbols) or smoking mothers (SM; closed symbols), expressed as: a) enhanced pause (Penh); and b) concentration of MCh inducing a 300\% increase in Penh ( $\mathrm{PC}_{300}$. c) Correlation of $\mathrm{PC}_{300}$ with airway smooth muscle layer. ${ }^{*}: \mathrm{p}<0.05 ;{ }^{* *}: \mathrm{p}<0.01 ;{ }^{* * *}: \mathrm{p}<0.001$; from subgroup analyses. ${ }^{*}: \mathrm{p}<0.001$ (SM effect) from linear regression analysis. a) HDM effect (NSM and SM groups) $p<0.05$ from linear regression analysis. b) HDM effect (NSM and SM groups): $p<0.001$ from linear regression analysis. c) rho $-0.62, p<0.001$. "HDM effect" indicates a difference between both PBS-exposed groups versus both HDMexposed groups. "SM effect" indicates a difference between both NSM groups versus both SM groups. associated $(\mathrm{p}<0.05)$. These three remodelling parameters explained $40 \%\left(\mathrm{R}^{2}\right)$ of the variability in PC 300 in a linear regression model. A model with only smooth muscle layer thickness accounted for $39 \%$ of the variability in PC 300 . Interestingly, eosinophils in lung tissue were also significantly correlated with PC300 and adding eosinophils to the first model increased the explained variability in PC 300 to $46 \%$, with a further increase to $59 \%$ when adding smoking during pregnancy to this model.

\section{DISCUSSION}

The present study has three important observations. First, maternal smoking during pregnancy increased airway smooth muscle layer thickness and collagen III deposition around the airways in offspring and increased HDM-induced goblet cell hyperplasia. Secondly, maternal smoking during pregnancy increased HDM-induced numbers of neutrophils and mast cells, and thirdly, maternal smoking increased MCh responsiveness in 10-week-old offspring.

The present study is the first to show that smoking during pregnancy, without subsequent postnatal exposure to tobacco smoke, increases and sustains remodelling in 10-week-old offspring. An additional new finding is that increased smooth muscle layer thickness is the best predictor of increased airway responsiveness in offspring from smoking mothers. Since contraction of airway smooth muscle upon antigen stimulation causes airway narrowing, the increase in smooth muscle found in offspring from smoking mothers may account for the increased $\mathrm{MCh}$ responsiveness that was observed. The finding that $\mathrm{MCh}$ responsiveness is linked to airway smooth muscle thickening in the airways is supported by SoUTHAM et al. [16], who found that (without introducing maternal smoking during pregnancy) persistent airway hyperresponsiveness is associated with increased smooth muscle area in mice exposed to HDM. In a study from ELLIOT et al. [17], however, the increase in acetylcholine chloride responsiveness in offspring from smoking guinea pig mothers was not associated with increased smooth muscle area, possibly because changes in smooth muscle layer thickness are not yet apparent 21 days after birth.

In mouse offspring exposed to maternal smoking in utero, $\mathrm{MCh}$ responsiveness was reportedly associated with a decreased presence of cyclic AMP in lung tissue [18]. This is of interest, since cyclic AMP is involved in relaxation of smooth muscle in the airway. Therefore, together with an increased smooth muscle area, other smooth muscle characteristics in the offspring exposed to maternal smoking during pregnancy may be a major contributor to airway hyperresponsiveness.

The effects of the in utero smoke-induced increase in airway smooth muscle contraction upon MCh challenge could even be enhanced by a decreased adaptive capacity of the airway wall caused by the increased collagen III deposition, as observed in the present study. Collagens are responsible for the compliance and structural integrity of the lung and increased collagen deposition, as seen in remodelled airways, is thought to render a stiffer airway. Interestingly, SEKHON et al. [19] have previously found that maternal nicotine exposure increased collagen III mRNA levels and protein expression in fetal monkey lungs, which was indeed associated with increased pulmonary resistance and decreased expiratory flows, probably due to stiffness of the airways. However, the latter study did not reveal 


\begin{tabular}{|c|c|c|c|c|c|c|}
\hline & \multicolumn{2}{|c|}{ NSM } & \multicolumn{2}{|c|}{ SM } & \multicolumn{2}{|c|}{ Linear regression analysis } \\
\hline Eosinophils volume \% & $0.05(0.03-0.07)$ & $0.36(0.05-0.43)^{\star *}$ & $0.05(0.04-0.07)$ & $0.29(0.18-0.65)^{\star \star \star}$ & $p<0.001$ & $p=0.431$ \\
\hline $\begin{array}{l}\text { Neutrophils } \mathrm{n} \text { per } \mathrm{mm}^{2} \\
\text { lung tissue }\end{array}$ & $181(134-245)$ & $220(177-293)$ & $163(116-207)$ & $269(181-354)^{\star \star}$ & $p=0.002$ & $p=0.986$ \\
\hline
\end{tabular}

Data are presented as median (interquartile range), unless otherwise stated. NSM: nonsmoking mother; SM: smoking mother; HDM: house dust mite. ${ }^{* *}$ : $<<0.01$; ${ }^{* \star *}$ $p<0.001$ from subgroup analysis (HDM versus PBS group).

whether these changes would remain throughout adult life or be reversible due to further lung development and/or maturation after birth. The present study indicates that effects of maternal smoking on collagen III deposition remain present even after a prolonged period of absence of smoke exposure.

Thus, both increased smooth muscle area and collagen III deposition may contribute to the observed increased $\mathrm{MCh}$ responsiveness in adult offspring exposed to smoke in utero.

The present study found a larger increase in HDM-induced goblet cells in offspring from smoking than from nonsmoking mothers. Goblet cell hyperplasia has been shown before to occur in response to active smoke exposure in animal models and COPD patients. The present report is the first to demonstrate increased goblet cell hyperplasia in response to maternal smoke exposure. So far, increased goblet cell numbers have been reported in asthma [20]; the main consequence of goblet cell hyperplasia is hypersecretion of mucin glycoproteins, causing mucous plugs that block the airways, contributing to increased airway hyperresponsiveness in these patients.

In addition to the effect of smoking during pregnancy on remodelling of the airways, numbers of HDM-induced neutrophils and mast cells were increased in offspring from smoking mothers but not in offspring from nonsmoking mothers. Several studies have shown that neutrophils are able to contribute to airway smooth muscle cell growth via production of elastase [21]. The mast cells present can also play an important role in proliferation of airway smooth muscle cells, collagen synthesis and hyperresponsiveness [22], mediated via release of, for example, tryptase, histamine and activin A. Although the increase in mast cell numbers in the present study was limited, these cells were mainly located in the airway wall. Thus, the combined increase in HDM induced mast cells and neutrophils, could underly changes in airway remodelling in HDM exposed offspring from smoking mothers. The mechanism behind the increase in neutrophils and mast cells, but not in eosinophils, IgE and Th2 cytokines, in association with maternal smoking during pregnancy remains unknown.

To further assess whether remodelling could predict MCh responsiveness, a linear regression model was used to show that smooth muscle layer thickness explained a considerable part of MCh responsiveness, independent of smoking during pregnancy. Interestingly, addition of numbers of lung tissue eosinophils to the model increased the explained variability of

\section{TABLE 2 Cytokines in lung tissue from offspring}

\begin{tabular}{|c|c|c|c|c|c|c|}
\hline \multirow[t]{2}{*}{ Cytokine pg $\cdot \mathrm{mL}^{-1}$} & \multicolumn{2}{|c|}{ NSM } & \multicolumn{2}{|c|}{ SM } & \multicolumn{2}{|c|}{ Linear regression analysis } \\
\hline & PBS & HDM & PBS & HDM & HDM effect & SM effect \\
\hline IL-2 & 229 (195-309) & $306(239-414)^{*}$ & 224 (187-285) & $292(242-330)^{*}$ & $p=0.002$ & $p=0.265$ \\
\hline IL-4 & $2.1(1.7-2.7)$ & $9.1(2.9-26.9)^{\star \star \star}$ & $1.9(1.7-1.9)$ & $7.0(6.0-21.4)^{\star * *}$ & $p<0.001$ & $p=0.986$ \\
\hline IL-5 & $12.5(9.9-17.8)$ & $17.8(10.6-26.7)$ & $8.6(8.6-14.2)$ & $13.9(7.3-28.4)$ & $p=0.028$ & $p=0.072$ \\
\hline IL-13 & $542(500-613)$ & $542(472-724)$ & $500(444-549)$ & $556(528-725)^{\star \star}$ & $p=0.014$ & $p=0.759$ \\
\hline IL-17 & $12.4(10.9-14.5)$ & $1018(83.9-3144)^{* * *}$ & $11.7(10.9-12.4)$ & $1096(160-2068)^{\star \star \star}$ & $p<0.001$ & $p=0.925$ \\
\hline IFN- $\gamma$ & $95.3(69.8-124.6)$ & $62.5(28.3-96.6)$ & $72.7(50.0-102.1)$ & $64.2(19.1-83.8)$ & $p=0.027$ & $p=0.302$ \\
\hline TNF & $14.2(11.8-16.2)$ & $15.4(11.8-19.3)$ & $12.2(10.7-13.7)$ & $15.7(13.7-19.2)^{\star \star *}$ & $p=0.012$ & $p=0.415$ \\
\hline Eotaxin & $1588(1376-1803)$ & $2222(1678-2475)^{\star \star}$ & $1450(1313-1637)$ & $1809(1527-2417)^{\star}$ & $p<0.001$ & $p=0.319$ \\
\hline
\end{tabular}

Data are presented as median (interquartile range), unless otherwise stated. NSM: nonsmoking mother; SM: smoking mother; HDM: house dust mite; IL: interleukin; IFN: interferon; TNF: tumour necrosis factor. ${ }^{*}: p<0.05$; ${ }^{* *}: p<0.01 ;{ }^{* *}: p<0.001$ from subgroup analysis (HDM versus PBS group). 
the model substantially. Thus, both increased smooth muscle area and allergic eosinophilic inflammation contributed independently to the severity of MCh responsiveness in adult mice. This may suggest that interventions to reduce airway hyperresponsiveness need to assess both features of asthma. Finally, the addition of smoking during pregnancy to the model also increased the explained variability substantially, indicating an independent effect of smoking during pregnancy on the severity of airway hyperresponsiveness. This shows that other factors affected by smoking during pregnancy play a role, like airway geometry or airway size due to changes in lung development in utero, or changes in innate immune responses, as proposed by NOAKEs et al. [9]. Nasal obstruction caused by intranasal HDM exposure could also be a factor inducing changes in Penh, thereby affecting the variability in PC300 explained by the different factors in the linear regression model. However, this effect is not fully explanatory since nasal obstruction cannot have affected effects of maternal smoking during pregnancy on Penh in PBS exposed offspring.

Besides the serious effects of smoking during pregnancy on lung function and structure in offspring, the present study provides new insights on the effects of HDM exposure with respect to cytokines and cell types involved in the inflammatory response. It was shown that, in particular, IL-17 levels strongly increased, which correlated with neutrophil and eosinophil influx and eotaxin production. This is in accordance with literature showing that IL-17 induces neutrophil recruitment [23] and is involved in eosinophil development [24] and eotaxin production [25]. Furthermore, support was found for some of the known effects of HDM exposure, such as increased eosinophils and goblet cells in lung tissue, increased IL-4, IL-5, IL-13 (previously investigated in bronchoalveolar lavage fluid and at mRNA level) and eotaxin in lung tissue, as well as increased (HDM-specific) serum IgE levels and remodelling of the lung [26-28]. Whether changes in cytokine levels are caused by changes in the levels in a specific compartment of the lung (e.g. airways and parenchyma) cannot be answered with the current approach using whole lung tissue.

In conclusion, these data indicate that smoking during pregnancy in mice induces several aspects of airway remodelling in adult mice offspring, which probably contribute to increased $\mathrm{MCh}$ responsiveness, even after a prolonged period without smoke exposure. Smoking during pregnancy induces remodelling, irrespective of allergen exposure but worsens the outcome of the allergic stimulus, resulting in the highest $\mathrm{MCh}$ responsiveness in HDM-exposed offspring from smoking mothers. The present study provided the opportunity to dissect the relative contribution of maternal smoking during pregnancy from the contribution of postnatal passive smoke exposure, showing long-lasting effects on lung structure and function in mice offspring.

The results support data from epidemiological studies on the association between maternal smoking and childhood or adolescent asthma. Moreover, the data provide suggestive evidence that, in particular, eosinophilic airway inflammation and increased airway smooth muscle area independently contribute to severity of airway hyperresponsiveness in asthma.

\section{ACKNOWLEDGEMENTS}

The authors acknowledge the help of P.A. Klok, C.A. Brandsma, M. Versluis, J.M. Vonk (all University Medical Center Groningen, University of Groningen, Groningen, the Netherlands) and the colleagues of the Central Animal Facility (University of Groningen).

\section{REFERENCES}

1 Pattenden S, Antova T, Neuberger M, et al. Parental smoking and children's respiratory health: independent effects of prenatal and postnatal exposure. Tob Control 2006; 15: 294-301.

2 Jaakkola JJ, Kosheleva AA, Katsnelson BA, Kuzmin SV, Privalova LI, Spengler JD. Prenatal and postnatal tobacco smoke exposure and respiratory health in Russian children. Respir Res 2006; 7: 48.

3 Moshammer H, Hoek G, Luttmann-Gibson $\mathrm{H}$, et al. Parental smoking and lung function in children: an international study. Am J Respir Crit Care Med 2006; 173: 1255-1263.

4 Gilliland FD, Berhane K, McConnell R, et al. Maternal smoking during pregnancy, environmental tobacco smoke exposure and childhood lung function. Thorax 2000; 55: 271-276.

5 Strachan DP, Butland BK, Anderson HR. Incidence and prognosis of asthma and wheezing illness from early childhood to age 33 in a national British cohort. BMJ 1996; 312: 1195-1199.

6 Gilliland FD, Islam T, Berhane K, et al. Regular smoking and asthma incidence in adolescents. Am J Respir Crit Care Med 2006; 174: 1094-1100.

7 Devereux G, Barker RN, Seaton A. Antenatal determinants of neonatal immune responses to allergens. Clin Exp Allergy 2002; 32: 43-50.

8 Atici A, Altintas D, Yuksel B, et al. Do parental smoking and history of allergy influence cord-serum IgE? Pediatr Allergy Immunol 1995; 6: 213-215.

9 Noakes PS, Hale J, Thomas R, Lane C, Devadason SG, Prescott SL. Maternal smoking is associated with impaired neonatal toll-like-receptor-mediated immune responses. Eur Respir J 2006; 28: 721-729.

10 Collins MH, Moessinger AC, Kleinerman J, et al. Fetal lung hypoplasia associated with maternal smoking: a morphometric analysis. Pediatr Res 1985; 19: 408-412.

11 Sekhon HS, Jia Y, Raab R, et al. Prenatal nicotine increases pulmonary $\alpha 7$ nicotinic receptor expression and alters fetal lung development in monkeys. J Clin Invest 1999; 103: 637-647.

12 Hafstrom O, Milerad J, Sundell HW. Prenatal nicotine exposure blunts the cardiorespiratory response to hypoxia in lambs. Am J Respir Crit Care Med 2002; 166: 1544-1549.

13 Elliot J, Vullermin P, Robinson P. Maternal cigarette smoking is associated with increased inner airway wall thickness in children who die from sudden infant death syndrome. Am J Respir Crit Care Med 1998; 158: 802-806.

14 van der Strate BW, Postma DS, Brandsma CA, et al. Cigarette smoke-induced emphysema: A role for the B cell? Am J Respir Crit Care Med 2006; 173: 751-758.

15 Melgert BN, Timens W, Kerstjens HA, et al. Effects of 4 months of smoking in mice with ovalbumin-induced airway inflammation. Clin Exp Allergy 2007; 37: 1798-1808. 
16 Southam DS, Ellis R, Wattie J, Inman MD. Components of airway hyperresponsiveness and their associations with inflammation and remodeling in mice. J Allergy Clin Immunol 2007; 119: 848-854.

17 Elliot J, Carroll N, Bosco M, McCrohan M, Robinson P. Increased airway responsiveness and decreased alveolar attachment points following in utero smoke exposure in the guinea pig. Am J Respir Crit Care Med 2001; 163: 140-144.

18 Singh SP, Barrett EG, Kalra R, et al. Prenatal cigarette smoke decreases lung cAMP and increases airway hyperresponsiveness. Am J Respir Crit Care Med 2003; 168: 342-347.

19 Sekhon HS, Keller JA, Proskocil BJ, Martin EL, Spindel ER. Maternal nicotine exposure upregulates collagen gene expression in fetal monkey lung. Association with $\alpha 7$ nicotinic acetylcholine receptors. Am J Respir Cell Mol Biol 2002; 26: 31-41.

20 Fahy JV. Remodeling of the airway epithelium in asthma. Am J Respir Crit Care Med 2001; 164: S46-S51.

21 Huang $\mathrm{CD}$, Chen $\mathrm{HH}$, Wang $\mathrm{CH}$, et al. Human neutrophilderived elastase induces airway smooth muscle cell proliferation. Life Sci 2004; 74: 2479-2492.

22 Yu M, Tsai M, Tam SY, Jones C, Zehnder J, Galli SJ. Mast cells can promote the development of multiple features of chronic asthma in mice. J Clin Invest 2006; 116: 1633-1641.
23 Oda N, Canelos PB, Essayan DM, Plunkett BA, Myers AC, Huang SK. Interleukin-17F induces pulmonary neutrophilia and amplifies antigen-induced allergic response. Am J Respir Crit Care Med 2005; 171: 12-18.

24 Kim MR, Manoukian R, Yeh R, et al. Transgenic overexpression of human IL-17E results in eosinophilia, Blymphocyte hyperplasia, and altered antibody production. Blood 2002; 100: 2330-2340.

25 Rahman MS, Yamasaki A, Yang J, Shan L, Halayko AJ, Gounni AS. IL-17A induces eotaxin-1/CC chemokine ligand 11 expression in human airway smooth muscle cells: role of MAPK (Erk1/2, JNK, and p38) pathways. J Immunol 2006; 177: 4064-4071.

26 Johnson JR, Wiley RE, Fattouh R, et al. Continuous exposure to house dust mite elicits chronic airway inflammation and structural remodeling. Am J Respir Crit Care Med 2004; 169: 378-385.

27 Cates EC, Fattouh R, Wattie J, et al. Intranasal exposure of mice to house dust mite elicits allergic airway inflammation via a GM-CSF-mediated mechanism. J Immunol 2004; 173: 6384-6392.

28 Simeone-Penney MC, Severgnini M, Tu P, et al. Airway epithelial STAT3 is required for allergic inflammation in a murine model of asthma. J Immunol 2007; 178: 6191-6199. 